This item is the archived peer-reviewed author-version of:

Workplace interventions to reduce HIV and TB stigma among health care workers : where do we go from here?

\title{
Reference:
}

Siegel Jacob, Yassi Annalee, Rau Asta, Buxton Jane A., Wouters Edwin, Engelbrecht Michelle C., Uebel Kerry E., Nophale Letshego E..- Workplace interventions to reduce HIV and TB stigma among health care workers : where do we go from here?

Global public health - ISSN 1744-1692 - (2015), p. 1-13

DOI: http://dx.doi.org/doi:10.1080/17441692.2015.1021365 


\section{Workplace Interventions to Reduce HIV and TB Stigma Among Healthcare Workers - Where do we go from here?}

Siegel $\mathrm{J}^{1}$, Yassi $\mathrm{A}^{1}$, Rau $\mathrm{A}^{2}$, Buxton $\mathrm{JA}^{1}$, Wouters ${ }^{2,3}$, Engelbrecht $\mathrm{MC}^{2}$, Uebel $\mathrm{KE}^{2,4}$, Nophale L $\mathrm{E}^{2,5}$.

${ }^{1}$ School of Population and Public Health, University of British Columbia, Canada;

${ }^{2}$ Centre for Health Systems Research \& Development, University of the Free State, South Africa;

${ }^{3}$ Centre for Longitudinal \& Life Course Studies, University of Antwerp, Belgium;

${ }^{4}$ Department of Internal Medicine, Faculty of Health Sciences, University of the Free State, Bloemfontein, South Africa;

${ }^{5}$ Provincial Occupational Health Unit, Free State Department of Health, South Africa

Jacob Siegel MPH

School of Population and Public Health

University of British Columbia

2206 East Mall Vancouver BC V6T 1Z3 Canada

tel: 604-644-1604

jsiegel@alumni.ubc.ca

(corresponding author)

Dr. Annalee Yassi MD MSc FRCPC

School of Population and Public Health

2206 East Mall Vancouver BC V6T 1Z3 Canada

tel: 604-916-6764

annalee.yassi@ubc.ca

Dr. Asta Rau PhD

Centre for Health Systems Research \& Development, University of the Free State PO Box 339 Bloemfontein South Africa 9300

tel: $+27(0) 514013342$

rauahm@ufs.ac.za

Dr. Jane A Buxton MBBs MHSc FRCPC

School of Population and Public Health

2206 East Mall Vancouver BC V6T 1Z3 Canada

tel: 604-827-4001

Prof. Edwin Wouters PhD

Research Centre for Longitudinal \& Life Course Studies, University of Antwerp

Sint-Jacobsstraat 2

B 2000 Antwerp Belgium 
tel: 003232655541

edwin.wouters@uantwerpen.be

Dr. Michelle C Engelbrecht PhD

Centre for Health Systems Research \& Development, University of the Free State PO Box 339 Bloemfontein South Africa 9300

tel: +27524013256

engelmc@ufs.ac.za

Dr. Kerry E Uebel MD PhD

Centre for Health Systems Research \& Development, University of the Free State PO Box 339 Bloemfontein South Africa 9300

tel: +27 514012181

uebelk@ufs.ac.za

Letshego E Nophale MSocSC

Provincial Occupational Health Unit

Department of Community Health

Faculty Of Health Sciences

University of the Free State

PO Box 339 Bloemfontein South Africa 9300

gngmlen@ufs.ac.za 


\title{
Workplace Interventions to Reduce HIV and TB Stigma Among Healthcare Workers- Where do we go from here?
}

\begin{abstract}
Fear of stigma and discrimination among healthcare workers in South African hospitals is thought to be a major factor in the high rates of HIV and tuberculosis infection experienced in the healthcare workforce. The aim of the current study is to inform the development of a stigma reduction intervention in the context of a large multicomponent trial. We analyzed relevant results of four feasibility studies conducted in the lead up to the trial. Our findings suggest that a stigma reduction campaign must address community and structural level drivers of stigma, in addition to individual level concerns, through a participatory and iterative approach. Importantly, stigma reduction must be embedded in the institutional management of healthcare workers but also be attentive to the localized needs of healthcare workers themselves.
\end{abstract}

Keywords: stigma reduction, HIV/AIDS, tuberculosis, healthcare workers, workplace interventions Word Count: 6746 


\section{Introduction}

The World Health Organization (WHO), International Labour Organization (ILO) and Joint United Nations Programme on HIV/AIDS (UNAIDS) issued guidelines in 2010 on improving Human Immunodeficiency Virus (HIV) and tuberculosis (TB) services for healthcare workers (HCWs) (World Health Organization \& International Labour Organization, 2010). To help ascertain how best to implement these guidelines, a large multicomponent cluster randomized controlled trial (RCT) was launched in South Africa within an international research collaboration to improve the health of HCWs globally, focusing on strengthening the use of hospital occupational health units (OHUs) for the prevention, diagnosis and treatment of HIV and TB among HCWs at hospitals in the Free State province (Yassi et al., 2014).

South Africa was the appropriate country in which to launch this study as HIV, TB and HIV/TB co-infection represent an enormous burden in the country. The number of incident TB cases in South Africa is among the highest in the world at 1\% (World Health Organization, 2013), while the rate of HIV amongst South Africa adults is 17.9\% (UNAIDS, 2013) and HIV prevalence in incident TB cases is 63\% (World Health Organization, 2013). In the Free State province of South Africa, public health facilities function in resource-constrained circumstances, with large patient numbers and over-crowded health facilities (RSA National Department of Health, 2011). There are severe shortages in human resources for health - not least due to the estimated $15.7 \%$ HIV prevalence among HCWs (Adams, Ehrlich, Ismail, Quail, \& Jeebhay, 2013) and the risk of contracting TB for HCWs nearly 4 times that of the general population (Baussano et al., 2011) which drives absenteeism, sick leave and attrition. 
In this context, the HIV/TB co-epidemic lays a double burden on human resources: HCWs have to 'cope with increased morbidity and mortality in [their] own ranks... [and] shoulder the impact of a rapidly increasing disease burden in the general population' (Lehmann \& Sanders, 2002, pg 127). This has prompted many health authorities to acknowledge that HIV, in addition to TB, among HCWs is an occupational health issue that needs to be managed in the workplace. Indeed, scholars continue to suggest that HCWs require increased access to HIV and TB services as part of an overall health systems strengthening strategy (Ncayiyana, 2004; World Health Organization \& International Labour Organization, 2010).

According to South African national policy, OHUs must provide a comprehensive standard level of service for HIV and TB, including education, prevention measures and on-site testing with appropriate referral to primary health care facilities for treatment (RSA Department of Public Service and Administration, 2008; South African National AIDS Council, 2012). In response to the high rates of TB among HCWs, this trial was designed to explore the added benefit of providing $\mathrm{HCWs}$ with the option of obtaining TB treatment and antiretroviral treatment (ART) at the OHU rather than outside the hospital at private or public facilities; it also aims to examine the benefit of rigorously implementing TB and other infection control measures to protect HCWs (Yassi et al., 2014).

Concerns regarding HIV and TB stigma are prevalent in HCWs (Baussano et al., 2011; Cianelli et al., 2011; Gounden \& Moodley, 2000) and are known to create a significant barrier for hospital staff to access and utilize HIV and TB services in general, whether at their workplace or at other facilities (de Vries, Galvin, Mhlanga, Cindzi, \& Dlamini, 2011; Namakhoma et al., 
2010; Uebel, Nash, \& Avalos, 2007). For instance, it has been shown that HCWs who work with people living with HIV experience stigma and discrimination from colleagues based on fear of infection and association with 'improper or immoral behaviour' (Ha, Chuc, Hien, Larsson, \& Pharris, 2013, pg 3). We therefore decided to include an HIV and TB stigma reduction component in the trial in order to specifically address stigma among HCWs, that is, stigma enacted by HCWs towards other HCWs as well towards themselves. We are also interested in ascertaining the extent to which addressing stigma could improve OHU utilization.

Stigma, as originally defined by Goffman (1963), and refined in more recent HIV literature (see for example Mahajan et al. (2008), Holzemer et al. (2007), and Castro \& Farmer (2005)) is a process of differentiation, othering and discrimination. Manifestations of external stigma include outward, negative attitudes, perceptions and behaviours targeted towards those with HIV and/or TB. Internal stigma includes negative beliefs that a person holds about themselves based on their lived experiences and their 'self-interpreted view of stigma' (Holzemer et al., 2007, pg 547; Kipp et al., 2011; Van Rie et al., 2008). Both types of stigma lead to low levels of job satisfaction, a reduction in the quality of life in the workplace, and ultimately poor health (Chirwa et al., 2009; Holzemer et al., 2007).

HIV and TB stigma intersects clinical and social modalities and its complex foundations call for complex interventions that are reflexive to evolving situations and target multiple domains of population health. Researchers have posited a number of critical recommendations for intervention research. First, interventions must address structural influences of stigma, such as removing workplace policies that perpetuate stigmatization (e.g. mandatory HIV or TB testing 
for employees or labeling beds of patients with HIV) (Stangl, Lloyd, Brady, Holland, \& Baral, 2013). Second, programs must place increased emphasis on upstream forces of power and inequality through a rights based approach (Parker \& Aggleton, 2003) (e.g. acknowledging the right of HCWs to be free of discrimination and marginalization and holding accountable hospital managers and policy makers to protect this right) (Gruskin, Bogecho, \& Ferguson, 2010; Kirkemann Boesen \& Martin, 2007). Third, interventions should utilize participatory and community based methods for program design and evaluation (e.g. involving HCWs in the direct design of a stigma reduction campaign) (Nyblade, Stangl, Weiss, \& Ashburn, 2009). Fourth, interventions should include a contact component whereby the target population is introduced to, and works together with, people living with HIV and TB in the development of stigma reduction projects or policies (Brown, Macintyre, \& Trujillo, 2003; Malachowski \& Kirsh, 2013) (supported further by mental health stigma research, see Corrigan, Morris, Michaels, Rafacz \& Rusch (2012)). Fifth, experiential evidence from various projects indicates that stigma is reduced when clinical capacity to test and treat HIV is increased (Castro \& Farmer, 2005; Uebel et al., 2007). These strategies work to extend the depth and breadth of the majority of current interventions and show promise in reducing stigma and ultimately reducing the burden of HIV and TB among HCWs.

This article describes the work we conducted to guide the development of an intervention designed to increase utilization of OHUs for HIV and TB services, reduce HIV and TB stigma among HCWs and ultimately affect its critical downstream effects on the health of HCWs. Feasibility studies of various types are needed when the literature is non-existent or inconclusive, or when more information is needed about the local context in order to design an effective 
intervention study (Bowen et al., 2009). Despite the wealth of knowledge available on stigma, many interventions continue to show little or no effect, and none have been conducted in the context of stigma by HCWs towards other HCWs. The pilot studies discussed in this paper were designed to bridge our current understanding of stigma in the realm of the healthcare workforce and to inform the development of a specialized intervention to combat HIV and TB stigma among HCWs.

\section{Methods}

Details of the trial methodology are available elsewhere (Yassi et al., 2014); in brief, the clinical component of the trial commenced in 2013 at all 27 public Free State hospitals with a staffed OHU (out of a total 30 public hospitals). Hospitals were matched by HCW population size and one of each pair randomly allocated to serve as an intervention site. OHUs in intervention sites offered full HIV and TB diagnosis and treatment to HCWs, while OHUs in comparison sites offered HIV and TB diagnosis, but referral for treatment elsewhere. Between 2011 and 2013, we conducted four studies to inform various aspects of the trial. Results that are relevant to stigma in the healthcare workplace and associated barriers to OHU utilization were drawn upon for this study. Other components of the trial remain in development pending full synthesis and discussion of these and other pilot studies with the various stakeholders.

\section{a. Stigma, Confidentiality and Barriers to use of OHUs}

A mixed-methods, non-random quota sampling study was carried out in three Free State hospitals, seeking to characterize occupational health and safety concerns and practices among HCWs to inform and serve as baseline for further work 
(Engelbrecht et al., in press). A self-administered questionnaire was completed by $978 \mathrm{HCWs}$ representing $20 \%$ of the total workforce at each hospital. This questionnaire included a section aimed at identifying factors relating to the uptake of HIV and TB services at hospital OHUs, including specific items pertaining to HIV stigma and confidentiality in the workplace. Questions were posed as agree/disagree, and proportions were calculated (Khan, 2013; Khan et al., 2014). In addition, thirty-eight participants were purposively selected to participate in 5 focus group sessions, which were conducted using a semi-structured schedule in order to contextualize the findings from this section of the questionnaires (Khan, 2013).

\section{b. Occupational Health Service Provision Tool}

A baseline assessment was conducted to ascertain the availability and training of OHU staff, availability of equipment and data management, and the full range of services provided. Two team members conducted individual interviews with occupational health nurses who worked at the OHUs at all 27 trial hospitals. A service provision survey was designed and used in these interviews in order to collect information on services available at OHUs for HIV and TB prevention, diagnosis, treatment and support. Pertinent to our study on stigma, the survey included questions on the opinion of OHU nurses on how often confidentiality is maintained at the OHU, if OHU nurses believe there is HIV and TB stigma in the workplace, and the perception of OHU nurses on the percentage of HCWs that use the OHU for HIV and TB services. Participants were asked to rate the occurrence of stigmatizing behaviours on a 4-point scale (never, sometimes, often, always) and responses were summarized by hospital.

c. Workplace HIV and TB Stigma Pilot Study 
Despite the importance of this issue, there are no validated questionnaires available to measure HIV and TB stigma among HCWs. The research team followed rigorous conceptual frameworks for the development of the stigma items, and also included questions on demographics, basic HIV and TB knowledge, confidentiality and disclosure in the workplace, and HIV and TB testing. A total of 41 individual items evaluated the multiple types of stigma among HCWs, and were posed as statements to which participants were asked to rate their agreement on a 4 point Likert scale (1 - strongly disagree to 4 - strongly agree) (Rau et al., 2013).

The pilot study was conducted at a regional hospital not included in the trial. Four field workers (including JS) personally recruited 220 participants with the assistance of hospital department managers so as to be representative of staff by profession (doctors, nurses, allied health professionals, administrative and support). Analysis included descriptive statistics of the stigma scores, and bivariate analyses of stigma, profession and HIV and TB knowledge level.

\section{d. Piloting HIV and TB Stigma Reduction Communication Tools}

Based on insights from health communication literature (see for example Glanz, Rimer \& Viswanath (2008)) and in line with Pauwels' study of HIV and TB health promotion materials in the Free State province (2005), the team conceptualized a stigma reduction campaign that would utilize core images and messages in several mediums such as posters, decals and other marketing materials in addition to communications from hospital managers to staff, promoting anti-stigma, anti-discrimination and assuring HCWs of the confidentiality of OHUs. Visual messaging images (see Figure 1) were designed to focus on the following attributes: Aesthetics, cultural appeal and appropriateness, literacy and 'Ubuntu' (community cohesion and togetherness). The 
messaging directly expresses the goals of the UNAIDS Getting to Zero campaign (zero stigma, zero new HIV/TB infections, zero HIV/TB deaths) (UNAIDS, 2010) which are also enshrined in South Africa's National Strategic Plan for HIV, STIs and TB, 2012-2016 (South African National AIDS Council, 2012); thus, the overall design aims for confluence and congruency between national goals and messages at the local level.

Eleven HCWs at five hospitals volunteered to participate in a study of the effectiveness of stigma reduction images. The messaging developed was piloted using a structured interview format in which respondents were asked to discuss their thoughts regarding each series. Responses were analyzed for common themes, and images and slogans that generated the most positive responses were modified, re-piloted and finalized for distribution.

\section{Results}

As summarized in Table 1, and expanded upon below, improvements in OHU services and reduction in HIV and TB stigma are necessary to support the health of HCWs. The findings underline the importance of stigma reduction interventions employing workplace campaigns targeting a wide range of approaches, including knowledge and awareness, policy change and support and appeal to the local sense of community and togetherness.

\section{a. Stigma, Confidentiality and Barriers to use of OHUs}

As noted by Khan et al. (2014), of the $978 \mathrm{HCWs}$ from the three hospitals where this questionnaire survey was conducted, $38.5 \%$ believed that there is HIV stigma in the workplace, and $38.9 \%$ stated that they believed that fear of lack of confidentiality was a reason for 
underutilization of the OHU for HIV counseling and testing by the healthcare workforce.

Overall, the reasons suggested for lack of use of the OHU for any service were: Not given enough time off during work day to visit OHU (18.4\%); concern for having to pay for services (8.7\%); and no point if antiretroviral medication is not provided at the clinic $(9.5 \%)$ (Khan, 2013). Results from focus groups indicated that HCWs are aware of how stigma is perpetuated in their workplace. Participants suggested that all HCWs should receive ongoing education on 1) specific regional and institutional policies regarding stigma and confidentiality, 2) the practical implications of living with HIV as it relates to working in a hospital, and 3) the impact of HIV on colleagues' mental health and wellbeing. As elaborated further by Khan et al. (2014) and Khan (2013), despite some apparent discordance between knowledge, beliefs and behaviours, HCWs expressed a desire for stronger organizational support to combat stigma in addition to addressing real or perceived breaches of confidentiality.

\title{
b. Occupational Health Service Provision Tool
}

Nurses in 9 of the 27 (33\%) Free State hospitals included in the trial reported that there was at least some TB stigma in the workplace. Nurses in $16(59 \%)$ of the sites reported that there was at least some HIV stigma in the workplace. Nurses in 13 (48\%) hospitals reported that confidentiality is always maintained at the OHU, with the remainder reporting 'often' or 'sometimes.' When asked what would encourage more hospital workers to utilize the OHU for HIV and/or TB services, nurses suggested education/awareness of HIV/TB and OHU services, as well as increasing capacity or service options (e.g. offering HIV medication) at the OHU.

\author{
c. Workplace HIV and TB Stigma Pilot Study
}


In the pilot study conducted in one hospital to develop a valid stigma questionnaire, $199 \mathrm{HCWs}$ completed questionnaires. We found that, overall, HIV and TB stigma was fairly low, with nearly all factors scoring below 2 (where 1 is lowest stigma and 4 is highest stigma). Bivariate analysis showed that stigma was negatively associated with both education and contact (knowing a colleague with HIV or TB) and that medical professionals reported observing less stigma than their non-medical professional counterparts. Respondents who reported standing up against stigmatizing behaviours were more likely to report: knowing their workplace confidentiality code of conduct; having been taught about the importance of protecting confidentiality in the workplace; and, knowing a colleague who has HIV. The latter was also associated with reporting higher perceptions of HIV stigma in the workplace by others and less HIV stigmatizing behaviours by the respondent.

\section{d. HIV and TB Stigma Messaging Pilot}

The images developed for this pilot were aimed at encouraging a sense of community among HCWs to promote the reduction of HIV and TB stigma. Four of the images piloted are shown in Figure 1. Analysis of responses revealed a number of common themes regarding the visual design and messaging of health promotion images. First, images and slogans must be 'catchy' and culturally appealing; for example, the use of shweshwe patterns, which are common across South Africa in traditional and modern fabrics, was very well received by many of the participants (the shweshwe pattern is represented in Figure 1, images A and C). Second, images must be clearly and directly related to the stigma reduction campaign. Some messaging series utilized imagery that was considered vague, such as a large 'zero' (to represent the UNAIDS Getting to Zero campaign mentioned above; see Figure 1, image A). Other images, such as the 
band aid in Figure 1, image B, utilized slogans that were not directly associated to the problem of stigma in the workplace. Although the band aid was seen as the connection between the working environment and health, the overall messaging was lost in the abstract nature of the slogan.

Third, messaging should be presented in the three languages commonly spoken in the Free State province (English, Afrikaans and SeSotho). This is critical for a multi-racial workforce;

furthermore, the simultaneous use of all three languages was also positively recognized by most participants, regardless of race, as further promoting the concept of 'Ubuntu' - community togetherness. The large hand in the 'stop' position (Figure 1, image C) was immediately associated with 'stopping' stigma and identified as a favourite among respondents. It utilizes a straightforward slogan that is reflected in the stop hand image itself. We also tested images to highlight the importance of maintaining confidentiality in the OHU; as was found among the stigma prevention images, those that reinforced the written message were regarded by respondents to be most effective (Figure 1, image D). Respondents appreciated the explicit association of the office files behind a lock, commenting that it served as a visual reminder of the importance of confidentiality in the workplace.

\section{Discussion and conclusion}

This article synthesized data from pilot and baseline studies we conducted to inform the development of an evidence-based HIV and TB stigma reduction campaign among HCWs. Synthesis studies have been conducted in similar contexts (Uebel, Guise, Georgeu, Colvin, \& Lewin, 2013) where systematic reviews were unavailable. In addition, the use of feasibility studies - the drawing of data from smaller studies aimed at collecting information on a wide variety of factors - in order to guide the design of complex interventions, has been 
acknowledged to be useful where the literature is otherwise unclear (Bowen et al., 2009). In this case, scholarly literature on how to design and launch an intervention to reduce HIV and TB stigma among HCWs, where stigma is directed not at patients but towards themselves or other HCWs, is essentially non-existent. As such, the approach described in this study was necessary.

The initial survey conducted in three large hospitals in the Free State province in 2011-2012 highlighted stigma and confidentiality concerns as potential barriers to uptake of HIV and TB services in OHUs. It is noteworthy that OHU nurses in 27 hospitals (which included two of the three hospitals in the stigma, confidentiality and barriers study) did not discuss stigma as an explicit barrier to OHU service utilization, but focused instead on improving services. Analyzing the overall qualitative and quantitative data we collected suggests that stigma is important, but that an equally important related issue is that the workforce as a whole may not be adequately aware of professional codes of conduct regarding confidentiality, nor of the resources and services available to protect their health and promote their well-being.

At the stigma questionnaire pilot study site, HIV and TB stigma levels were considered to be moderately low, although present across all occupational groups. While HIV and TB stigma among HCWs towards other HCWs has not previously been measured, our results are consistent with previous studies that have found similarly low levels of HIV and TB stigma directed at HCWs by patients, or by HCWs directed at patients (Coreil, Lauzardo, \& Heurtelou, 2012; Feyissa, Abebe, Girma, \& Woldie, 2012; Uys et al., 2009). It is important to note that as the categorization and construct of stigma scales varies widely across studies, there is no benchmark of HIV and TB stigma in the workplace or elsewhere with which to compare our results and 
accurate comparisons are only possible from a qualitative standpoint. Regardless of the magnitude, we feel that stigma is a problem worth attempting to address.

Strategies for HIV and TB stigma reduction must be aligned with theoretically grounded or empirically proven approaches. As published research about HIV and TB stigma reduction in the healthcare workplace interventions is essentially non-existent, it is useful to examine studies related to mental health stigma interventions and overall workplace health studies, as well as literature on stigma in other settings in order to derive guidance on developing interventions. Such literature strongly suggests that individual level interventions on their own do not have the capacity to impact the underlying social processes and factors that enable stigmatization. Stangl et al. (2013) have most recently suggested the need for HIV stigma interventions to target multiple domains of stigma as well as multiple levels of the socio-ecology model. Communication strategies at the individual level primarily include educational workshops and skills training, while at the community level, strategies aim to target the social and cultural norms, values and attitudes that encourage or discourage stigmatizing behaviours. At the structural/social level, strategies focus on the policies and regulations of government and institutions that support healthy and equitable practices (UNAIDS \& World Health Organization, n.d.). In addressing these multiple levels, we intend to utilize social and behaviour change communication (SBCC), which is widely accepted among public health practitioners and specifically employs multiple levels of communication to promote changes in health behaviour (Christofides, Nieuwoudt, Usdin, Goldstein, \& Fonn, 2013; UNAIDS \& World Health Organization, n.d.). 
Workplace interventions in the areas of HIV and mental health stigma recognize the value of participatory approaches - those in which employees at all levels (i.e. front line, administration and management) are involved in the design, implementation and evaluation of activities aimed at addressing multiple aspects of workplace environment and health, such as mental wellbeing, physical environment, organizational support and structural conditions (Kobayashi, Kaneyoshi, Yokota, \& Kawakami, 2008). Li, Guan, Liang, Lin \& Wu (2013) utilized the Diffusion of Innovation theory, originally developed by Rogers (1983), to create a Popular Opinion Leaders (POL) HIV stigma intervention; the program's success suggests that locally developed interventions that are attuned to institutional social networks and feature POLs prominently may be quite useful. The participatory approach has been implemented in the workforce beyond the scope of HIV and TB stigma. Henning et al. (2009) emphasize the value of engaging teams of workers in the iterative design of workplace interventions, and Punnet (2009) noted that participatory programs that instill a sense of empowerment are likely to bolster program effectiveness; they called for better integration of 'workplace health-promotion programs [with] attention to the work environment, especially in light of recent evidence that work organization influences so-called lifestyle or health behaviours' (p. 16). Arts-based interventions, such as the participatory theatre productions discussed in Quinlan (2009) and Gray, Fitch, Labrecque \& Greenberg (2003) have similarly demonstrated success in changing attitudes and behaviours among HCWs in workplace bullying and patient care. The work of Punnet, et al. (2009) is particularly applicable, and attention should be paid to highly participatory techniques, such as participatory theatre used to assist HCWs in addressing workplace bullying (Quinlan, 2009). While there are differences between bullying and stigmatization, certainly successful 
interventions in the healthcare workplace related to the former are worth considering in planning interventions to combat HIV stigma towards HCWs.

In developing a model for stigma towards people with mental illness, Corrigan, Markowitz, Watson, Rowan \& Ann (2003) build on Weiner's (1993) Attribution Theory, which attempts to associate people's perceptions of the cause of illness, their emotional response and ultimately discriminatory behaviours. Attribution Theory provides a causal mechanism of stigmatizing attitudes and behaviours; for example, fear of contagion (of HIV or TB) can aggravate stigmatizing behaviours, while familiarity/contact (knowing someone with HIV) can mitigate such behaviours. The idea of familiarity, for instance, is something built into the SBCC poster slogans we described, such as 'Let's stop stigma'; with the 'Ubuntu' and community togetherness messages - communicating that 'we, as HCWs, are all in this together' - are particularly important to diminish perceived gaps between self and other.

The merging of the paradigms of workplace health promotion and workplace health and safety has long been advocated as an effective strategy (Yassi, 2005). Novel intervention strategies must address embedded attitudes among HCWs, discriminatory regulations at the facility and regional level (Nyblade et al., 2009), and the rights of people living with HIV and/or TB (Gruskin et al., 2013). In addition, active involvement of HCWs, their representatives, as well as workers living with HIV, as called for by the WHO-ILO-UNAIDS guidelines, would be consistent with the evidence we found in this study. Within this approach, integrating a lively, flexible, relevant and highly responsive stigma reduction campaign into multi-level and comprehensive interventions that combine occupational health with health promotion stands a 
good chance of successfully reducing HIV and TB stigma among HCWs, thereby supporting increased utilization of HIV and TB services and ultimately improving the health and wellbeing of the healthcare workforce.

\section{Acknowledgements}

The team wishes to thank the many health workers in the Free State province, as well as colleagues from University of British Columbia, South Africa Department of Health, the South African National Institute for Occupational Health, The Free State Department of Health, University of the Free State, and the World Health Organization and International Labour Office who participated in, and indeed led, the various feasibility studies and initiatives described in this article.

\section{Funding}

This work was supported by the Canadian Institutes of Health Research (CIHR) under grant ROH-115212, 'Promoting Health Equity by Addressing the Needs of Health Workers: A Collaborative, International Research Program.'

\section{Conflict of Interest}

The authors declare there is no conflict of interest. 
References

Adams, S., Ehrlich, R., Ismail, N., Quail, Z., \& Jeebhay, M. (2013). Occupational Health Challenges Facing the Department of Health: Protecting employees against tuberculosis and caring for former mineworkers with occupational health disease. In South African Health Review 2012/13 (pp. 67-82). Durban: Health Systems Trust.

Baussano, I., Nunn, P., Williams, B., Pivetta, E., Bugiani, M., \& Scano, F. (2011). Tuberculosis among health care workers. Emerging Infectious Diseases, 17(3), 488-94. doi:10.3201/eid1703.100947

Bowen, D. J., Kreuter, M., Spring, B., Cofta-Woerpel, L., Linnan, L., Weiner, D., ... Fernandez, M. (2009). How we design feasibility studies. American Journal of Preventive Medicine, 36(5), 452-7. doi:10.1016/j.amepre.2009.02.002

Brown, L., Macintyre, K., \& Trujillo, L. (2003). Interventions to reduce HIV/AIDS stigma: what have we learned? AIDS Education and Prevention, 15(1), 49-69. doi:10.1521/aeap.15.1.49.23844

Castro, A., \& Farmer, P. (2005). Understanding and addressing AIDS-related stigma: from anthropological theory to clinical practice in Haiti. American Journal of Public Health, 95(1), 53-9. doi:10.2105/AJPH.2003.028563

Chirwa, M. L., Greeff, M., Kohi, T. W., Naidoo, J. R., Makoae, L. N., Dlamini, P. S., ... Holzemer, W. L. (2009). HIV stigma and nurse job satisfaction in five African countries. Journal of the Association of Nurses in AIDS Care, 20(1), 14-21. doi:10.1016/j.jana.2008.10.001

Christofides, N. J., Nieuwoudt, S., Usdin, S., Goldstein, S., \& Fonn, S. (2013). A South African university-practitioner partnership to strengthen capacity in social and behaviour change communication. Global Health Action, 6, 19300. doi:10.3402/gha.v6i0.19300

Cianelli, R., Ferrer, L., Norr, K. F., Mccreary, L., Irarrázabal, L., \& Miner, S. (2011). Stigma Related to HIV among Community Health Workers in Chile. Stigma Research and Action, 1(1), 3-10. doi:10.5463/SRA.v1i1.11

Coreil, J., Lauzardo, M., \& Heurtelou, M. (2012). Anticipated tuberculosis stigma among health professionals and Haitian patients in South Florida. J Health Care Poor Underserved, 23(2), 636-650. doi:10.1353/hpu.2012.0046

Corrigan, P. W., Markowitz, F. E., Watson, A., Rowan, D., \& Ann, M. (2003). An Attribution Model of Public Discrimination towards Persons with Mental Illness. Journal of Health and Social Behavior, 44(2), 162-179. 
Corrigan, P. W., Morris, S. B., Michaels, P. J., Rafacz, J. D., \& Rusch, N. (2012). Challenging the Public Stigma of Mental Illness: A Meta-Analysis of Outcome Studies. Psychiatric Services, 63(10). doi:10.1176/appi.ps.005292011

De Vries, D. H., Galvin, S., Mhlanga, M., Cindzi, B., \& Dlamini, T. (2011). "Othering" the health worker: self-stigmatization of HIV/AIDS care among health workers in Swaziland. Journal of the International AIDS Society, 14(1), 60. doi:10.1186/1758-2652-14-60

Engelbrecht, M., Yassi, A., Spiegel, J., van Rensburg, A., O'Hara, L., Bryce, E., ... Rau, A. (in press). Tuberculosis and blood-borne infectious diseases: Workplace conditions and practices of healthcare workers at three public hospitals in the Free State. Southern Africa Journal of Epidemiology and Infection.

Feyissa, G. T., Abebe, L., Girma, E., \& Woldie, M. (2012). Validation of an HIV-related stigma scale among health care providers in a resource-poor Ethiopian setting. Journal of Multidisciplinary Healthcare, 5, 97-113. doi:10.2147/JMDH.S29789

Glanz, K., Rimer, B. K., \& Viswanath, K. (Eds.). (2008). Health behavior and health education. Theory, research and practice (4th ed., p. 590). San Francisco, CA: Jossey-Bass.

Goffman, E. (1963). Stigma: notes on the management of spoiled identity. Garden City, NY: Anchor Books.

Gounden, Y. P., \& Moodley, J. (2000). Exposure to human immunodeficiency virus among healthcare workers in South Africa. International Journal of Gynecology \& Obstetrics, 69(3), 265-270. doi:10.1016/S0020-7292(00)00207-1

Gray, R. E., Fitch, M. I., Labrecque, M., \& Greenberg, M. (2003). Reactions of health

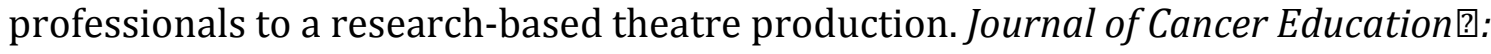
The Official Journal of the American Association for Cancer Education, 18(4), 223-9. doi:10.1207/s15430154jce1804_10

Gruskin, S., Bogecho, D., \& Ferguson, L. (2010). "Rights-based approaches” to health policies and programs: articulations, ambiguities, and assessment. Journal of Public Health Policy, 31(2), 129-45. doi:10.1057/jphp.2010.7

Gruskin, S., Safreed-Harmon, K., Ezer, T., Gathumbi, A., Cohen, J., \& Kameri-Mbote, P. (2013). Access to justice: evaluating law, health and human rights programmes in Kenya. Journal of the International AIDS Society, 16(Suppl 2), 18726. doi:10.7448/IAS.16.3.18726

Ha, P. N., Chuc, N. T. K., Hien, H. T., Larsson, M., \& Pharris, A. (2013). HIV-related stigma: Impact on healthcare workers in Vietnam. Global Public Health, 8(Suppl 1), S61-S74. doi:10.1080/17441692.2013.799217 
Henning, R., Warren, N., Robertson, M., Faghri, P., Cherniack, M., \& CPH-NEW Research Team. (2009). Workplace health protection and promotion through participatory ergonomics: an integrated approach. Public Health Reports, 124(Suppl 1), 26-35.

Holzemer, W. L., Uys, L., Makoae, L., Stewart, A., Phetlhu, R., Dlamini, P. S., ... Naidoo, J. (2007). A conceptual model of HIV/AIDS stigma from five African countries. Journal of Advanced Nursing, 58(6), 541-51. doi:10.1111/j.1365-2648.2007.04244.x

Khan, R. (2013). Stigma and Confidentiality as Barriers to Uptake of HIV Counseling and Testing for Health Workers in 3 Public Hospitals in Free State Province, South Africa: A Mixed-Methods Study. University of British Columbia.

Khan, R., Yassi, A., Engelbrechtc, M., Nophale, L., van Rensburg, A., \& Spiegel, J. (2014). Barriers to uptake of HIV counseling and testing for health workers in 3 public hospitals in Free State Province, South Africa. AIDS Care. Advance online publication. doi:10.1080/09540121.2014.951308

Kipp, A. M., Pungrassami, P., Nilmanat, K., Sengupta, S., Poole, C., Strauss, R. P., ... Van Rie, A. (2011). Socio-demographic and AIDS-related factors associated with tuberculosis stigma in southern Thailand: a quantitative, cross-sectional study of stigma among patients with TB and healthy community members. BMC Public Health, 11, 675. doi:10.1186/1471-2458-11-675

Kirkemann Boesen, J., \& Martin, T. (2007). Applying a rights-based approach: An inspirational guide for civil society. Copenhagen.

Kobayashi, Y., Kaneyoshi, A., Yokota, A., \& Kawakami, N. (2008). Effects of a worker participatory program for improving work environments on job stressors and mental health among workers: a controlled trial. Journal of Occupational Health, 50(6), 45570. doi:10.1539/joh.L7166

Lehmann, U., \& Sanders, D. (2002). Human resource development: human resources. South African Health Review, 119-133.

Li, L., Guan, J., Liang, L.-J., Lin, C., \& Wu, Z. (2013). Popular Opinion Leader intervention for HIV stigma reduction in health care settings. AIDS Education and Prevention, 25(4), 327-35. doi:10.1521/aeap.2013.25.4.327

Mahajan, A. P., Sayles, J. N., Patel, V. a, Remien, R. H., Sawires, S. R., Ortiz, D. J., ... Coates, T. J. (2008). Stigma in the HIV/AIDS epidemic: a review of the literature and recommendations for the way forward. AIDS, 22 Suppl 2, S67-79.

doi:10.1097/01.aids.0000327438.13291.62

Malachowski, C., \& Kirsh, B. (2013). Workplace antistigma initiatives: a scoping study. Psychiatric Services, 64(7), 694-702. doi:10.1176/appi.ps.201200409 
Namakhoma, I., Bongololo, G., Bello, G., Nyirenda, L., Phoya, A., Phiri, S., ... Obermeyer, C. M. (2010). Negotiating multiple barriers: health workers' access to counselling, testing and treatment in Malawi. AIDS Care, 22 Suppl 1(November 2013), 68-76. doi:10.1080/09540121003607241

Ncayiyana, D. J. (2004). Doctors and nurses with HIV and AIDS in sub-Saharan Africa. BMJ, 329(7466), 584-5. doi:10.1136/bmj.329.7466.584

Nyblade, L., Stangl, A., Weiss, E., \& Ashburn, K. (2009). Combating HIV stigma in health care settings: what works? Journal of the International AIDS Society, 12, 15.

doi:10.1186/1758-2652-12-15

Parker, R., \& Aggleton, P. (2003). HIV and AIDS-related stigma and discrimination: a conceptual framework and implications for action. Social Science \& Medicine, 57(1), 13-24. doi:10.1016/S0277-9536(02)00304-0

Pauwels, L. (2005). Posters, billboards and grassroots media relating to TB and AIDS in the Free State and Lesotho. Acta Academia Supplementum, 1, 337-353.

Punnet, L., Cherniak, M., Henning, R., Morse, T., Faghri, P., \& CPH-NEW Research Team. (2009). A Conceptual Framework for Integrating Workplace Health Promotion and Occupational Ergonomics Programs. Public Health Reports, 124(Suppl 1), 16-25.

Quinlan, E. (2009). New action research techniques: Using Participatory Theatre with health care workers. Action Research, 8(2), 117-133. doi:10.1177/1476750309335204

Rau, A., Uebel, K. E., Wouters, E., Engelbrecht, M., Buxton, J., Siegel, J., ... Yassi, A. (2013). HIV and TB stigma among health care workers: A pilot survey in a large Free State hospital. In Free State Provincial Health Research Day. Bloemfontein, South Africa.

RSA Department of Public Service and Administration. (2008). Employee Health and Wellness Strategic Framework for the Public Service. Pretoria.

RSA National Department of Health. (2011). Human Resources for Health for South Africa, 2030. Draft Strategy for the Health Sector 2012/2013 - 2016-2017; Consultation Document. Pretoria.

Siegel, J. (2013). Stigma Campaign Image Pilot Progress Report. Retrieved from http://ghrp.ubc.ca/?attachment_id=4875

South African National AIDS Council. (2012). National Strategic Plan on HIV, STIs and TB 2012-2016.

Stangl, A. L., Lloyd, J. K., Brady, L. M., Holland, C. E., \& Baral, S. (2013). A systematic review of interventions to reduce HIV-related stigma and discrimination from 2002 to 2013: 
how far have we come? Journal of the International AIDS Society, 16(Suppl 2). doi:10.7448/IAS.16.3.18734

Uebel, K. E., Guise, A., Georgeu, D., Colvin, C., \& Lewin, S. (2013). Integrating HIV care into nurse-led primary health care services in South Africa: a synthesis of three linked qualitative studies. BMC Health Services Research, 13, 171. doi:10.1186/1472-6963-13171

Uebel, K. E., Nash, J., \& Avalos, A. (2007). Caring for the caregivers: models of HIV/AIDS care and treatment provision for health care workers in Southern Africa. The Journal of Infectious Diseases, 196(Suppl 3), S500-4. doi:10.1086/521113

UNAIDS. (2010). Getting to Zero: 2011-2015 Strategy.

UNAIDS. (2013). South Africa HIV and AIDS Estimates. Retrieved November 12, 2013, from http://www.unaids.org/en/regionscountries/countries/southafrica/

UNAIDS, \& World Health Organization. (n.d.). Social Change Communication. Technical Guidance for Global Fund Proposals. Retrieved January 15, 2014, from http://www.who.int/hiv/pub/toolkits/2-3a_SocialChangeCommunication_09EN.pdf

Uys, L. R., Holzemer, W. L., Chirwa, M. L., Dlamini, P. S., Greeff, M., Kohi, T. W., ... Naidoo, J. R. (2009). The development and validation of the HIV/AIDS Stigma Instrument - Nurse (HASI-N). AIDS Care, 21(2), 150-9. doi:10.1080/09540120801982889

Van Rie, A., Sengupta, S., Pungrassami, P., Balthip, Q., Choonuan, S., Kasetjaroen, Y., ... Chongsuvivatwong, V. (2008). Measuring stigma associated with tuberculosis and HIV/AIDS in southern Thailand: exploratory and confirmatory factor analyses of two new scales. Tropical Medicine \& International Health, 13(1), 21-30. doi:10.1111/j.1365-3156.2007.01971.x

World Health Organization. (2013). Global tuberculosis report 2013.

World Health Organization, \& International Labour Organization. (2010). Joint WHO / ILO policy guidelines on improving health worker access to prevention, treatment and care services for HIV and TB. Geneva.

Yassi, A. (2005). Health promotion in the workplace - the merging of the paradigms. Methods of Information in Medicine, 44(2), 278-84.

Yassi, A., Hara, L. M. O., Engelbrecht, M. C., Uebel, K., Nophale, L. E., Bryce, E. A., ... Spiegel, J. M. (2014). Considerations for preparing a randomized population health intervention trial: lessons from a South African-Canadian partnership to improve the health of health workers. Global Health Action, 7(23594). 
Table 1: Findings and Implications of feasibility studies

\section{Data source Findings}

Stigma,

confidentiality

and barriers to

use of OHUs

(2011-2012)

b Occupational

Health Service

Provision Tool

(2013)

c

$$
\text { Workplace HIV }
$$

and TB Stigma

Pilot Study

d $\quad$ HIV and TB

Stigma

Messaging Pilot

(2013)

Implications for Stigma Reduction

Intervention

Concerns of HIV and TB stigma, as

well as fear of breach of

confidentiality at the $\mathrm{OHU}$ were high

among HCWs. Many respondents

expressed the need for increased

education and organizational support.

OHU nurses believe HIV and TB

stigma is common in their facilities

but that confidentiality for the most

part, is well maintained by $\mathrm{OHU}$

professionals. $\mathrm{OH}$ nurses cited the

need for increasing capacity of $\mathrm{OHU}$

to provide services, along with

awareness of the confidential services

available to $\mathrm{HCWs}$, as a requirement

to boost utilization.

HIV and TB stigma among HCWs

was rated relatively low. Education,

professional cadre and knowing

someone with HIV were associated

with lowered stigmatizing behaviours.

Messages that were inclusive, direct

and utilized unique imagery were

favoured by HCWs.
Knowledge and awareness continue to be important factors in reducing stigma; definitive codes of conduct regarding confidentiality at the $\mathrm{OHU}$ must be publicized and maintained; buy-in and support from facility and regional managers necessary for broad level improvements in stigma and confidentiality. Improvements in OHU services and reduction in HIV and TB stigma are necessary to support the health of HCWs. There may be discordance between perceived and actual breaches of confidentiality among OHU nurses, which would merit being addressed in a workplace campaign.

Stigma reduction interventions may target specific professions; campaigns that include education, structural support and contact components will show most success.

Promotion materials must be locally relevant, appealing, and mobilize traditional notions of being part of a community. 


\section{List of Figures}

Figure 1: Examples of images designed for stigma reduction communication campaign. A: "Getting to Zero." B: "Caring for HCWs." C: "Let's Stop Stigma.” D: "Keeping Confidentiality." 